\title{
Justiça distributiva no mapeamento inicial dos atendimentos pré-hospitalares de um país em desenvolvimento
}

DOI: $10.46919 / \operatorname{archv2n3-034~}$

Recebimento dos originais: 01/01/2021

Aceitação para publicação: 31/03/2021

\section{Diego Santiago Montandon}

Doutor. Grupo de Estudos e Pesquisas em Comunicação no Processo de Enfermagem - GEPECOPEn da Escola de Enfermagem de Ribeirão Preto da Universidade de São Paulo - USP. Av. Bandeirantes, 3900 Campus Universitário - Bairro Monte Alegre Ribeirão Preto - SP - Brazil

E-mail: montandonds@alumni.usp.br

\section{Luiz Carlos Souza de Oliveira}

Mestre. Grupo de Estudos e Pesquisas em Comunicação no Processo de Enfermagem - GEPECOPEn da Escola de Enfermagem de Ribeirão Preto da Universidade de São Paulo - USP. Av. Bandeirantes, 3900 Campus Universitário - Bairro Monte Alegre Ribeirão Preto - SP - Brazil

E-mail: luizoliveiraenf@gmail.com

\section{Leonardo Batista Pedroso}

Doutor. Grupo de Estudos Agronômicos Aplicados ao Sudoeste Goiano do Instituto Federal de Educação, Ciência e Tecnologia Goiano - IFGoiano, Campus Morrinhos. Rodovia BR153, KM633. Zona rRural

Morrinhos - GO - Brazil

E-mail: pedroso88@msn.com

\section{Laysi da Silva Zacarias}

Mestre. Grupo de Estudos Agronômicos Aplicados ao Sudoeste Goiano do Instituto Federal de Educação, Ciência e Tecnologia Goiano - IFGoiano, Campus Morrinhos, Brasil. C - Núcleo de Estudos e Pesquisa em Cultura Jurídica e Atlântico Negro - Maré e do Centro de Estudos em Desigualdade e Discriminação CEDD, ambos da Universidade de Brásilia - UNB. Brasil. Campus Universitário Darcy Ribeiro. Asa

Norte - Brasília/DF - Brazil

E-mail: laysizacarias@alumni.usp.br

\section{João Pedro Resende Castro}

Mestre. Grupo de Estudos e Pesquisas em Comunicação no Processo de Enfermagem - GEPECOPEn da Escola de Enfermagem de Ribeirão Preto da Universidade de São Paulo - USP. Av. Bandeirantes, 3900 Campus Universitário - Bairro Monte Alegre Ribeirão Preto - SP - Brazil

E-mail: jprcastro@usp.br

\section{Simone de Godoy}

Doutor. Líder do Grupo de Estudos e Pesquisas em Comunicação no Processo de Enfermagem GEPECOPEn da Escola de Enfermagem de Ribeirão Preto da Universidade de São Paulo - USP. Av. Bandeirantes, 3900 Campus Universitário - Bairro Monte Alegre Ribeirão Preto - SP - Brazil

E-mail: sig@eerp.usp.br

\section{RESUMO}

A cidade de Ribeirão Preto no interior do Estado de São Paulo no Brasil tem a mais de 20 anos atendimento pré-hospitalar de urgência pública e gratuita, mesmo assim os recursos disponíveis ainda são insuficientes para atender adequadamente a demanda da população. Por isso é necessário analisar se existe justiça 
distributiva, aos moldes aristotélicos, no geoprocessamento destes atendimentos, com a finalidade de avaliar o principio da equidade ao acesso neste serviço de saúde. Método: estudo transversal, incluindo 41.389 ocorrências de urgência pré-hospitalar de 2014, cujas variáveis permitiram a construção de um mapa para avaliar a distribuição espacial dos atendimentos no município. Resultado: Houve predomínio de atendimentos clínicos do tipo cardiológicos, maior número de atendimentos nos menores distritos urbanos e equivalência de socorro diante da variável sexo. A analise do mapeamento comprova aglomeração dos atendimentos nas regiões centrais. Conclusão: Os arranjos espaciais dos atendimentos estão aglomerados na região central da cidade, portanto não evidenciou-se justiça distributiva pelo ponto de vista aristotélico.

Palavras Chaves: Justiça Social, Georreferênciamento, Atendimento Pré-hospitalar de Urgência, Ética na Enfermagem.

\begin{abstract}
The city of Ribeirão Preto, in the interior of the state of São Paulo in Brazil, has had free public pre-hospital emergency care for more than 20 years, even though the available resources are still insufficient to adequately meet the demand of the population. Therefore, it is necessary to analyze whether there is Aristotelian distributive justice in the geoprocessing of these services, in order to evaluate the principle of equity of access to this health service. Method: cross-sectional study, including 41,389 pre-hospital emergency occurrences in 2014, whose variables allowed the construction of a map to assess the spatial distribution of care in the city. Results: There was a predominance of cardiology clinical care, more care in the smallest urban districts and equivalence of help in relation to the variable gender. The mapping analysis proves the agglomeration of care in central regions. Conclusion: The spatial arrangements of care are agglomerated in the central region of the city, therefore distributive justice by the Aristotelian point of view was not evidenced.
\end{abstract}

Key words: Social Justice, Georeferencing, Pre-hospital Emergency Care, Nursing Ethics.

\title{
1 INTRODUÇÃO
}

$\mathrm{O}$ atendimento pré-hospitalar (APH) configura-se como uma forma de atenção à saúde direcionada a casos de ocorrências súbitas e externas às instituições de saúde, com o interesse de ofertar adequada resposta diante de convocações de socorro $^{(1)}$, pode estar relacionada desde uma orientação por telefone, até o envio de viaturas terrestres, aéreas ou náuticas ao encontro da vítima, para minimizar possíveis sequelas e proteger a vida ${ }^{(2)}$.

As ocorrências de APH estão relacionadas com causas clínicas e traumáticas, respectivamente segunda e terceira maiores causas de mortalidade ${ }^{(3)}$. Por isso que o sistema de saúde brasileiro investe neste tipo de atenção por intermédio do Serviço de Atendimento Móvel de Urgência (SAMU-192) ${ }^{(4)}$, cuja atividade atual é pública e gratuita no Brasil se estendendo a 926 cidades, com cobertura populacional de $44,6 \%{ }^{(5)}$.

O município de Ribeirão Preto no interior do Estado de São Paulo no Brasil, disponibiliza APH público desde 1996 e foi uma das primeiras cidades brasileiras a aderir ao SAMU-192 ${ }^{(6)}$. Em 2013 inaugurou seu núcleo administrativo dos APH's e começou a utilizar um gerenciador eletrônico para 
auxiliar o controle dos dados das chamadas de socorro, esta organização justifica o notório desenvolvimento em recursos para atendimento de urgências e emergências desta região na América Latina ${ }^{(7)}$. Mesmo com esta estrutura, a demanda social desta região ainda é maior que a disponibilidade da assistência e evidenciam-se elevadas taxas de mortalidade e mobilidade relacionadas a ocorrências clínicas e traumáticas $^{(7)(8)}$.

Diante desta realidade, emerge a necessidade de avaliar a equidade na alocação dos recursos de urgência pré-hospitalar para a sociedade, a fim de verificar as condições de acesso a este serviço público $^{(9)(10)}$. Deste modo, é importante realizar reflexões à luz da justiça distributiva, principio filosófico encabeçado por Aristóteles na qual é analisado se os recursos sociais são igualmente disponibilizados à população com direito a usufruir destes benefícios ${ }^{(11)(12)}$.

Para tal é importante considerar o advento das geotecnologias, que por intermédio do geoprocessamento utiliza mapas para analisar os eventos relacionados à saúde com suas distribuições de ocorrências epidemiológicas associadas ao espaço territorial e ao perfil socioeconômico da população investigada ${ }^{(13)}$.

Este trabalho tem o interesse de saber se a distribuição espacial inicial dos atendimentos préhospitalares de urgência no município de Ribeirão Preto ocorre de forma equânime. Com o objetivo de analisar sob a ótica da justiça distributiva o geoprocessamento dos APHs realizados inicialmente com auxílio de um gerenciador eletrônico.

\section{MÉTODOS}

\subsection{DESENHO DO ESTUDO E CONTEXTO (SETTING)}

Este é um estudo transversal ${ }^{(14)}$, realizado com 41.348 atendimentos prestados pelo SAMU-192 na cidade de Ribeirão Preto, interior do Estado de São Paulo, Brasil. O município tem uma população total de 658.059 habitantes e é dividido em cinco distritos urbanos de saúde: central, leste, norte, oeste e sul ${ }^{(15)}$.

Neste município ocorreu pela primeira vez na América Latina, triagens por telefone de forma organizada e sistematizada através do serviço público de atendimento pré-hospitalar local, que conta atualmente com 221 profissionais, duas viaturas de unidades de suporte avançado (USA) - equipe de APH composta por enfermeiro, médico e socorrista, destinada a casos com risco eminente de vida - e treze unidades de suporte básico (USB) - composto por profissionais de nível médio para atender casos menos complexos. Desde 2013, estas ambulâncias ficam alocadas em unidades de saúde dos distritos urbanos e são acionadas com auxílio de um gerenciador eletrônico que avalia por geoprocessamento o local do pedido de socorro e a proximidade das viaturas para que o despachante de ambulâncias tenha dados atuais e seguros para tomada de decisão. 


\subsection{PARTICIPANTES}

Foram avaliados os APHs do SAMU-192 desta localidade, que provocaram deslocamento de viaturas para assistência emergencial ocorridos em 2014. Os critérios de exclusão adotados estão relacionados aos casos de trote telefônicos, atendimentos resolvidos por telefone e transporte de pacientes entre instituições de saúde. A seguir o fluxograma de seleção dos casos:

Figura1: Fluxograma de seleção dos casos para este estudo. Ribeirão Preto - SP, Brasil. 2021.

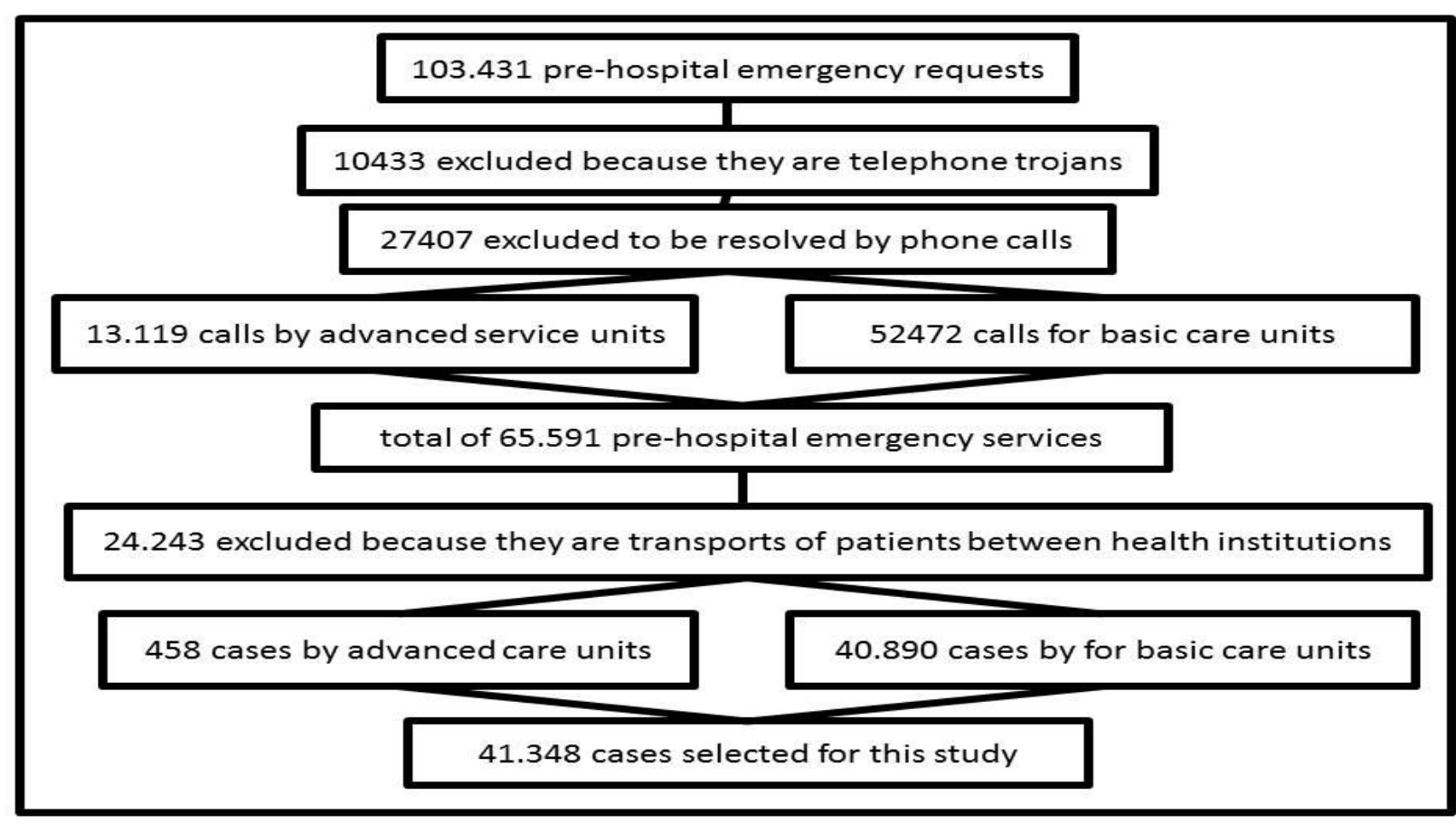

Fontes de dados/ Mensuração e variáreis

O local de estudo conta com um gerenciador eletrônico para auxiliar a triagem de emergência por telefone, no qual o despachante de ambulâncias digita os dados do atendimento relacionados com o endereço da ocorrência, sexo da vítima, queixa informada e observações especiais. A partir disso, o programa verifica por um sistema de geoprocessamento as viaturas mais próximas do local informado e estima o tempo necessário para o atendimento deste socorro, com estas informações o despachante de ambulâncias toma a decisão sobre o envio do atendimento pré-hospitalar. Caso haja o deslocamento de uma esquipe de atendimento pré-hospitalar, o gerenciador eletrônico armazena os dados relacionados com o tempo da ligação, tempo para acionamento das equipes de socorro, tempo de chegada da equipe no local. Ao finalizar o socorro, a equipe deve informar qual foi o tipo de atendimento e o desfecho da vítima para que o gerenciador eletrônico finalize o acompanhamento do caso. 


\subsection{CONTROLE DE VIÉS}

Com o interesse de analisar o impacto inicial da utilização do gerenciador eletrônico na justiça distributiva destes casos, optou-se por avaliar o ano de 2014, ano em que a equipe de despachantes de ambulâncias já estavam devidamente treinados para utilizar esta tecnologia de apoio na triagem por telefone dos pedidos de socorro.

\subsection{TAMANHO DO ESTUDO}

Este estudo foi realizado por uma amostra de conveniência e o emprego deste tipo de análise permitiu representar a distribuição espacial dos APHs por intermédio de um mapa, no qual é definindo a localização de cada evento selecionado e permite verificar visualmente se estão disseminados homogeneamente no município, ou ao contrário, concentram-se em alguns locais em detrimento doutros.

O mapa tem como base o sistema viário da cidade para possibilitar identificar as regiões urbanas e rurais, está divido entre as cincos regiões de saúde, cuja densidade de atendimentos da USB está representado pela tonalidade da coloração em cada subdivisão, as assistências prestadas pela USA configuram pontos no mapa, permitindo avaliar se há diferença entre as distribuições das equipes básicas.

Após as analises estatísticas de correlação houve uma abordagem sob a ótica Aristotélica de justiça distributiva dos APH's. Esta base filosófica de raciocínio foi escolhida por ser o alicerce ideológico do atual sistema democrático da localidade estudada, no qual se acredita que "toda virtude é gerada e deve ser destruída pelas mesmas causas e pelos mesmos meios"(16).

\subsection{VARIÁVEIS QUANTITATIVAS}

Os pesquisadores tiveram acesso as variáveis por uma planilha eletrônica disponibilizada pelo SAMU-192, foram analisadas as porcentagem de missings de cada dado, considerando viáveis para utilização nesta pesquisa as variáveis com menos de $5 \%$ de informações não preenchidas. Após analisar a viabilidade das variáveis, utilizou-se o sexo das vítimas, feminino e masculino; equipes de socorro, USA e USB; tipo de atendimento, clínico, traumático e suas especificações; distrito urbano de saúde da localidade, central, leste, norte, oeste e sul; longitude e latitude utilizados no mapa.

\subsection{MÉTODOS ESTATÍSTICOS}

Estes dados foram organizados, havendo calculo de sua prevalência, posteriormente foram processadas pelo software Qgis 2.8, que é um sistema de informações geográficas de utilização fácil, livre e licenciado no Brasil, criado para desenvolver mapas com informações ambientais e realizar analises de bioestatística espacial que difere da estatística tradicional por focalizar explicitamente a localização 
geográfica, considerando a importância de seu arranjo na análise e interpretação de resultados em saúde (17).

Para verificar se existe um padrão espacial de distribuição dos atendimentos da USA foi utilizado a técnica de Scan e das USB a estimativa de Kernel. Estes modelos geoestatísticos determinam se as variáveis no mapa estão distribuídas aleatoriamente, regularmente ou estão aglomerados em regiões específicas. Estes testes podem ser aferidos pelo índice global de Moran (I), que analisa a distância de cada evento ao centro do mapa e considera um padrão aglomerado quando tem a menor distância média entre pontos com grande desvio padrão, com I entre 0 e -1; a distribuição é regular quando existe uma grande distância média entre os pontos e pequeno desvio padrão, com I entre 0 e 1; e é considerada aleatória com distância média entre pontos e grande desvio padrão, com $\mathrm{I}=0^{(18)}$.

\subsection{QUESTÃO ÉTICA}

Por se tratar de estudo que utilizou em suas etapas, a análise de informações institucionais de seres humanos, o projeto foi submetido e aprovado pela Comissão de Avaliação de Projetos de Pesquisa da Secretaria Municipal de Saúde de Ribeirão Preto e ao clivo do Comitê de Ética em Pesquisa da Escola de Enfermagem de Ribeirão da Universidade de São Paulo com o Protocolo CAAE: 53039816.0.0000.5393.

\section{RESULTADOS}

Foram avaliados 41.348 atendimentos de urgência pré-hospitalar que acarretaram o deslocamento das viaturas USA $(n=458)$ e USB $(n=40890)$. A tabela I apresenta as frequências e porcentagens categorizadas dos casos.

Tabela 1: Prevalência dos atendimentos pré-hospitalares de urgência $(n=41348)$ quanto ao sexo, tipo de atendimento, distrito urbano de saúde e equipe responsável. Ribeirão Preto - SP, Brasil. 2021.

\begin{tabular}{|c|c|c|c|c|c|c|}
\hline \multirow[t]{2}{*}{$\begin{array}{l}\text { Variáveis } \\
\end{array}$} & \multicolumn{2}{|c|}{ USB $(n=40890)$} & \multicolumn{2}{|c|}{ USA $(n=458)$} & \multicolumn{2}{|c|}{ Total $(n=41348)$} \\
\hline & $\mathbf{N}$ & $\%$ & $\mathbf{N}$ & $\%$ & $\mathbf{N}$ & $\%$ \\
\hline \multicolumn{7}{|l|}{ Sexo } \\
\hline Masculino & 20581 & 50,3 & 292 & 63,8 & 20873 & 50,5 \\
\hline Feminino & 20309 & 49,7 & 166 & 36,2 & 20475 & 49,5 \\
\hline \multicolumn{7}{|c|}{ Tipo de Atendimento } \\
\hline Clinico & 25779 & 63.0 & 228 & 49,7 & 26007 & 62,8 \\
\hline Cardiológico & 16940 & 41,4 & 177 & 38,6 & 17117 & 41,4 \\
\hline Neurológico & 2786 & 6,8 & 3 & 0,7 & 2789 & 6,7 \\
\hline Obstétrico & 2363 & 5,7 & 3 & 0,7 & 2366 & 5,7 \\
\hline Psiquiátrico & 2124 & 5,2 & 10 & 2,2 & 2134 & 5,1 \\
\hline Pediatria & 1487 & 3,6 & 0 & 0,0 & 1487 & 3,6 \\
\hline Gastrointestinal & 1095 & 2,7 & 17 & 3,7 & 1112 & 2,6 \\
\hline Oncológico & 381 & 0,9 & 35 & 7,6 & 416 & 1,0 \\
\hline Oftalmológico & 90 & 0,2 & 0 & 0,0 & 90 & 0,2 \\
\hline Traumático & 15111 & 37,0 & 230 & 50,3 & 15341 & 37,2 \\
\hline Trânsito & 5476 & 13,3 & 209 & 45,6 & 4228 & 13,7 \\
\hline
\end{tabular}




\begin{tabular}{lcccccc}
\hline \multicolumn{1}{c}{ Queda } & 3566 & 8,7 & 3 & 0,7 & 3569 & 8,6 \\
$\quad$ Ortopédico & 2556 & 6,3 & 0 & 0,0 & 2556 & 6,2 \\
$\quad$ Perfuro-contundente & 1756 & 4,3 & 1 & 0,2 & 1757 & 4,2 \\
Otorrinolaringologia & 256 & 0,6 & 0 & 0,0 & 256 & 0,6 \\
$\quad$ Cirurgia cervical & 14 & 0,0 & 0 & 0,0 & 14 & 0,0 \\
$\quad$ Distrito urbano & & & & & & \\
Oeste & 10277 & 25,1 & 70 & 15,3 & 10347 & 25,0 \\
Norte & 8611 & 21 & 48 & 10,5 & 8632 & 20,8 \\
Central & 8056 & 19,7 & 126 & 27,5 & 8182 & 19,7 \\
Sul & 7464 & 18,3 & 76 & 16,6 & 7540 & 18.2 \\
Leste & 6482 & 15,9 & 138 & 30,1 & 6620 & 16,0 \\
\hline
\end{tabular}

Legenda: USB - Unidade de Suporte Básico; USA - Unidade de Suporte Avançado.

O geoprocessamento de cada caso permitiu a construção de um mapa, cujos atendimentos pela USA obteve $\mathrm{I}=-0,4$ e os realizados pelas USB com $\mathrm{I}=-0,9$, ou seja, ambos com distribuição espacial aglomerada.

O mapa apresenta o Estado de São Paulo no Brasil e a município de realização do estudo neste estado. A cidade é representada com a sua divisão entre os distritos de saúde cujos atendimentos de suporte avançado (n=458) são pontos no mapa, os atendimentos por USB são representados por porcentagens nas áreas urbanas de forma proporcional a intensidade dos atendimentos, quanto mais atendimentos houver na região do mapa, mais escuro aparece.

Figura 2: Mapa dos atendimentos pré-hospitalares de urgência divididos pelos distritos urbanos. Ribeirão Preto - SP, Brasil. 2021.
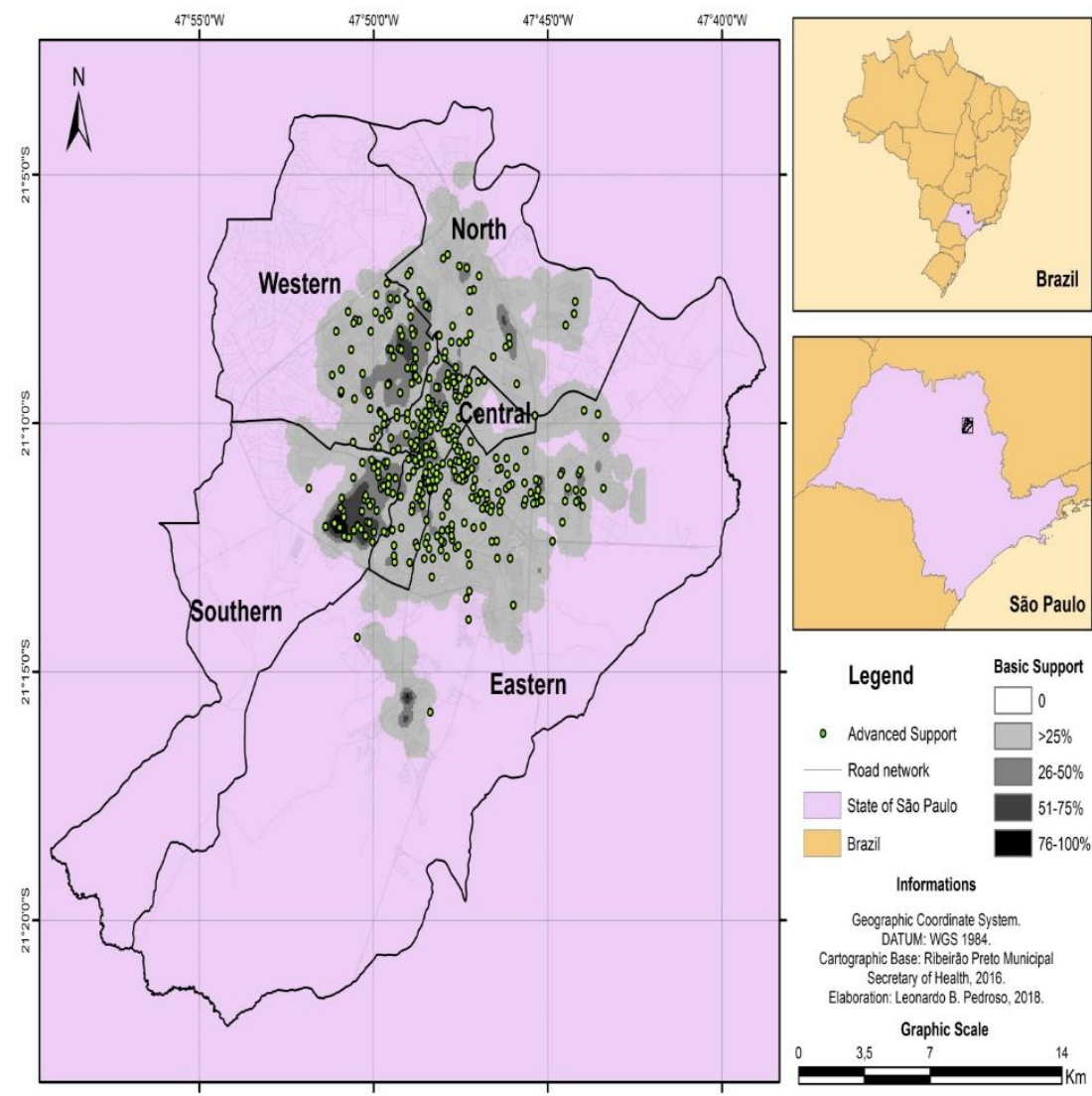


\section{DISCUSSÃO}

\subsection{ACHADOS PRINCIPAIS}

Foi identificado que a distribuição espacial dos APH's estão aglomerados na região central da cidade. Houve predomínio de atendimentos clínicos com destaque a afecções cardiológicas, maior número de atendimentos nos menores distritos urbanos e equivalência de socorro na variável sexo.

\subsection{INTERPRETAÇÕES DOS RESULTADOS}

As centralizações dos APH’s estão relacionadas com as características desta região da cidade, onde existe intenso transito de veículos e pessoas, com expressivo contingente de edifícios comerciais. Um estudo descritivo dos geoprocessamento dos atendimentos do SAMU - 192 em uma capital do nordeste brasileiro, também destaca maior ocorrência de casos em locais com grande fluxo de automóveis, maior contingente de pessoas e regiões comerciais, por alocarem momentaneamente grande número de pessoas aumentando

Os atendimentos do tipo clínicos - cardiológicos configuraram isoladamente maior prevalência que a soma de todos os traumáticos. Este fato ocorreu devido a aglomeração dos atendimentos serem em bairros centrais com habitantes majoritariamente idosos ${ }^{(20)}$ e consequentemente com maior risco de moléstias súbitas cardiovasculares, que de acordo com o Guidelines da American Heart Association devem ser abordadas imediatamente com $\mathrm{APH}^{(21)}$.

A mesma lógica de raciocínio aplica-se para justificar o fato de os menores distritos do município, Oeste, Norte e Central, obterem os maiores índices de APH's, pois são localidades com importante participação na mobilidade urbana, maior densidade demográfica de idosos, com expressivo comércio e portanto, são regiões com características simulares. O próprio poder executivo da cidade, afirma que a sua divisão em cinco distritos foi realizada para alocar localidades com semelhanças dentro do mesmo contexto e assim proporcionar condições especificas de atenção à saúde e de acesso aos serviços pela população ${ }^{(22)}$.

Quanto ao sexo, outros estudos também demonstram uma equivalência entre os atendimentos de urgência entre homens e mulheres, tendo em vista que o sexo masculino ainda tem dificuldade em aderir a programas de promoção da saúde e prevenção de agravos ${ }^{(3)}$ (8), justificando também sua maior prevalência entre os atendimentos de emergência realizados pela USA, enquanto o sexo feminino conquista diariamente maior ascensão social, lhes proporcionado também mais fatores de risco a problemas de saúde súbitos ${ }^{(4)(9)}$ (10).

Ao determinar que o arranjo espacial dos APH's desta cidade estão aglomeradas na região central, é possível afirmar que esta distribuição é injusta, de acordo com a visão Aristotélica, que considera justiça em dois âmbitos os corretivos e os distributivos, esta segunda consiste propriamente em disponibilizar igualmente os direitos públicos entre os que obtêm iguais deveres ${ }^{(11)(12)}$. Ao descrever este princípio 
filosófico para os resultados encontrados, observa-se que todas as pessoas que se encontram no município estudado tem o direito de serem atendidas pelo SAMU-192 e que para tal precisam estar em situação de urgência e entrar em contato com o serviço.

Neste sentido, apontam-se três premissas: 1 - Os habitantes das regiões periféricas da localidade não precisam assistência do SAMU-192; 2 - As convocações de socorro da periferia da cidade são ignoradas pelo serviço de saúde; 3 - Mesmo precisando de assistência do SAMU-192, a população não solicita seu auxílio.

Não é possível verificar qual a localidade das ligações de solicitação de socorro, mesmo assim nas periferias da cidade, principalmente nos distritos norte, oeste e sul, existe rodovias, comercio local, maiores índices de atropelamento, tentativas de homicídio por arma de fogo, estrupo vulnerável e lesão corporal por agressão ${ }^{(23)}$, perfazendo potenciais de necessidade de assistência pré-hospitalar.

Portanto é importante verificar se as solicitações de socorro advindas de regiões periféricas da cidade são ignoradas ou não chegam a ser solicitadas. Ambas as causas possibilitam afirmar que existem fatores que dificultam o acesso de pessoas alocadas em regiões periféricas ao APH disponibilizado pelo SAMU-192 e que a utilização inicial do gerenciador eletrônico para apoiar o despachante de atendimentos na tomada de decisão não é eficaz para distribuir justamente os APH’s.

\subsection{LIMITAÇÕES E DESFECHOS}

Este estudo não permitiu determinar a causa de poucos atendimentos na periferia da cidade estudada, nem se a utilização do gerenciador eletrônico melhorou a justiça distributiva deste tipo de assistência à saúde, mas disponibiliza dados iniciais da equidade de acesso a este bem público e apresenta justificativa para haver um acompanhamento prospectivo do georeferenciamento dos APH's para melhorias ao acesso de toda a população local ao SAMU-192.

\section{CONCLUSÃO}

A distribuição espacial dos atendimentos pré-hospitalares de urgência na localidade estudada não ocorre de forma justa do ponto de vista Aristotélico, pois a analise inicial dos APH's realizados com auxílio de uma gerenciador eletrônico tem aglomeração na região central do município em detrimento das porções periféricas, comportando-se de forma não equânime e universal.

Faz-se necessária a realização de análises prospectivas da implementação desta tecnologia com vista a garantir uma distribuição justa dos APH's com melhora significativa da assistência pré-hospitalar nesta região. 


\section{AGRADECIMENTOS}

À Coordenação de Aperfeiçoamento de Pessoal de Nível Superior (CAPES), pelo financiamento do estudo e ao Prof. Dr. Oméro Benedicto Poli Neto pelo direcionamento na apresentação destes resultados. 


\section{REFERÊNCIAS}

1. Franklin TA, Da Silva Santos HC, Santos Junior JA, Vilela ABA. Characterization of service of a pre-hospital care service to older persons involved in fall / Caracterização do atendimento de um serviço pré-hospitalar a idosos envolvidos em queda. Rev Pesqui Cuid é Fundam Online [Internet]. 2018;10(1):62. Available from: http://seer.unirio.br/index.php/cuidadofundamental/article/view/5977

2. Garlet ER, Lima MAD, Santos JLG, Marques GQ. Work organization of a health team in attending the user in urgency and emergency situations Portuguese]. Texto Context Enferm [Internet]. 2009; 18(2):266-72. Available from: http://search.ebscohost.com/login.aspx?direct=true \&db=cin20\&AN=2010402711\&lang=es\&site=ehostlive

3. BRASIL. Morbidade hospitalar do SUS por causas externas - por local de internação. 2018. p. acesso em 05 mar. 2018. Disponível em: http://tabn.

4. Ibiapino MK, Couto VBM, Sampaio BP, Souza RAR de, Padoin FA, Salomão IS. Serviço de atendimento móvel de urgência: epidemiologia do trauma no atendimento pré-hospitalar. Rev da Fac Ciências Médicas Sorocaba [Internet]. 2017;19(2):72. Available from: https://revistas.pucsp.br/index.php/RFCMS/article/view/30805

5. BRASIL $\mathrm{M}$ da S. Informações do SAMU. Brasil [Internet]. $2018 . \quad$ p. http://samu.saude.sc.gov.br/index.php/samu-br/abra.

6. Lopes SLB, Fernandes RJ. Uma breve revisão do atendimento médico pré-hospitalar. Medicina (B Aires) [Internet]. 1999;381-7. Available from: http://revista.fmrp.usp.br/1999/vol32n4/uma_breve_revisao_atendimento_medico_pre_hospitalar.pdf

7. Coelho MF, Chaves LDP, Anselmi ML, Hayashida M, Dos Santos CB. Análise dos aspectos organizacionais de um serviço de urgencias clínicas: estudo em um hospital geral do município de Ribeirão Preto, SP, Brasil. Rev Latino-Am Enferm. 2010;18(4):1-9.

8. Barbosa BMB, Monteiro RA, Sparano LF, Bareiro RFN, Passos ADC, Engel EE. Incidence and causes of lower-limb amputations in the city of Ribeirão Preto from 1985 to 2008: evaluation of the medical records from 3,274 cases. Rev Bras Epidemiol [Internet]. 2016;19(2):317-25. Available from: http://www.scielo.br/scielo.php?script=sci_arttext\&pid=S1415-790X2016000200317\&lng=en\&tlng=en

9. Almeida PMV de, Dell'Acqua MCQ, Cyrino CMS, Juliani CMCM, Palhares V de C, Pavelqueires S. Analysis of services provided by SAMU 192: Mobile component of the urgency and emergency care network. Esc Anna Nery - Rev Enferm [Internet]. 2016;20(2). Available from: http://www.gnresearch.org/doi/10.5935/1414-8145.20160039

10. Mendonça MFS de, Silva AP de SC, Castro CCL de. Análise espacial dos acidentes de trânsito urbano atendidos pelo Serviço de Atendimento Móvel de Urgência: um recorte no espaço e no tempo. Rev Bras Epidemiol [Internet]. 2017;20(4):727-41. Available from: http://www.scielo.br/scielo.php?script=sci_arttext\&pid=S1415-790X2017000400727\&lng=pt\&tlng=pt

11. Fortes PA de C. Orientações bioéticas de justiça distributiva aplicada às ações e aos sistemas de saúde. Rev Bioética. 2008;16(1):25-39. 
12. Ribeiro CD. Justiça social e equidade em saúde: Uma abordagem centrada nos funcionamentos. Saude e Soc. 2015;24(4):1109-18.

13. Alvares J, Bonini D. geoprocessamento, análise espacial, saúde pública. Rev UNINGÁ. 2010;26:189-97.

14. Denise F Polit; Cheryl Tatano Beck. Nursing research: generating and assessing evidence for nursing practice. 10 . Wolters Kluwer Health, editor. Philadelphia; 2017.

15. Elizabete Pagotti da Fonseca RI, Christiane Rodrigues Barbosa MI, Rossetti Ferreira III B. High prevalence of enteroparasites in children from. Rev Bras Enferm Brazil Rev Bras Enferm [Internet] [Internet]. 2017;7070(33):566-71566. Available from: http://dx.doi.org/10.1590/0034-7167-2016-0059

16. Eduardo CB Bittar. A justiça em Aristoteles. III. São Paulo: Leya; 2017. 163 p.

17. Da Silva RG et al. Sistema de Informação Geográfica no mapeamento de arboviroses no Território de Desenvolvimento Entre Rios, Estado do Piauí, Brasil. Archives of Health. 2020; 1(3):111-118.

18. Albert Skaba D, Sá Carvalho M, Barcellos C, Martins PC, Luiza Terron S. Geoprocessamento dos dados da saúde: o tratamento dos endereços. Rev Saude Publica [Internet]. 2004;20(6):1753-6. Available from: $89102003000400015 \& \operatorname{lng}=$ en\&nrm=iso

http://www.scielosp.org/scielo.php?script=sci_arttext\&pid=S0034-

19. Cabral AP de S, Souza WV de. Serviço de Atendimento Móvel de Urgência (SAMU): análise da demanda e sua distribuição espacial em uma cidade do Nordeste brasileiro. Rev Bras Epidemiol [Internet]. 2008;11(4):530-40. Available from: http://www.scielo.br/scielo.php?script=sci_arttext\&pid=S1415$790 X 2008000400002 \& \operatorname{lng}=\mathrm{en} \& \mathrm{nrm}=$ iso

20. BRASIL M da S. Bairros com mais idosos em Ribeirão Preto - SP [Internet]. 2010 [cited 2018 Apr 4]. p. 2. Available from: http://populacao.net.br/bairros-com-mais-idosos-ribeirao-preto_sp.html

21. Gerhard-Herman MD, Gornik HL, Barrett C, Barshes NR, Corriere MA, Drachman DE, et al. 2016 AHA/ACC guideline on the management of patients with lower extremity peripheral artery disease: Executive Summary: A report of the American college of cardiology/American Heart Association task force on clinical practice guidelines. Vol. 135, Circulation. 2017. 686-725 p.

22. Secretaria Municipal de Saúde de Ribeirão Preto. Distritos de Saúde de Ribeirão Preto [Internet]. 2013 [cited 2018 Apr 3]. Available from: https://www.ribeiraopreto.sp.gov.br/ssaude/i16saude.php

23. Secretaria Estadual de Segurança Pública de São-Paulo. Dados Estatisticos de 2014 em Ribeirão Preto - SP [Internet]. 2015 [cited 2018 Apr 2]. Available from: http://www.ssp.sp.gov.br/Estatistica/Mapas.aspx 